\title{
REFLECTION SYMMETRY PROPERTIES OF GENERALIZED FRACTIONAL DERIVATIVES
}

\author{
Malgorzata Klimek, Maria Lupa \\ Institute of Mathematics, Czestochowa University of Technology, Poland \\ mklimek@im.pcz.pl,maria.lupa@im.pcz.pl
}

\begin{abstract}
In this paper we study the properties of generalized fractional derivatives (GFDs) with respect to the reflection mapping in finite intervals. We introduce symmetric and antisymmetric derivatives in a given interval and a split of arbitrary function into [J]projections - parts with well-defined reflection symmetry properties. The main result are representation formulas for the symmetric and anti-symmetric GFDs of order $\alpha \in(0,1)$ which allow us to reduce the operators defined in the interval $[a, b]$ to the ones given in arbitrarily short subintervals.
\end{abstract}

\section{Introduction}

In our previous papers $[1,2]$, we investigated the reflection symmetry in fractional calculus. It appeared that the reflection operator which turns the left-sided fractional derivatives into the right-sided ones allows us to reduce the symmetric and anti-symmetric combinations of these operators to non-local derivatives determined in arbitrarily short subintervals. We also demonstrated that in some cases this property of fractional derivatives leads to a new phenomenon in fractional variational calculus - some of the Euler-Lagrange equations can be localized in subintervals of the main time-interval.

Fractional variational calculus, first introduced by Riewe in [3, 4], was developed by Agrawal in [5], Klimek in [6,7] and studied in many papers (compare [8-20] and the references given therein). In principle it leads to complicated integrodifferential equations which in contrast to the one-sided fractional differential equations cannot be easily and explicitly solved. This is the reason why a localization (if demonstrated in the general case) would be useful in the development of numerical and exact methods of solving fractional Euler-Lagrange equations.

In the present paper we consider the reflection symmetry in generalized fractional calculus introduced by Agrawal in [21]. In the definition of generalized fractional derivatives (GFDs), the non-local component is based on an arbitrary kernel. Let us point out that the first generalized operators of this type were defined in papers [22-24], where the kernel is a Mittag-Leffler function. The GFDs with such kernels have been recently applied in a study of wave equations and string vibrations $[25,26]$. Thus, the question arises whether in this general fractional calculus 
the operators have similar properties with respect to reflections in finite intervals. The results enclosed in this paper confirm that in the case, where the kernel of the non-local part is invariant with respect to translations, we can define the symmetric and anti-symmetric GFDs and represent them in the form of operators determined on arbitrarily short subintervals of the initial time-interval $[a, b]$.

\section{Preliminaries}

In this section we recall the basic definitions from generalized fractional calculus introduced in [21]. In the standard fractional calculus non-local differential operators are compositions of integer order derivatives and certain integral operators based on kernels - power functions. In the generalized fractional calculus the derivatives are similarly constructed, but the kernels are arbitrary functions defined in $[a, b]$ for which the integrals, given below, exist.

Definition 1.1. Let $\alpha>0$. The operator $K_{P}^{\alpha}$ of order $\alpha$ is given by the formula

$$
K_{P}^{\alpha} f(t)=p \int_{a}^{t} k_{\alpha}(t, \tau) f(\tau) d \tau+p \int_{t}^{b} k_{\alpha}(\tau, t) f(\tau) d \tau,
$$

where $a<t<b, P=(a, t, b, p, q)$ is a parameter set (called $P$-set), $k_{\alpha}(t, \tau)$ is a the kernel which may depend on parameter $\alpha$, and the parameters $p$ and $q$ are two real numbers.

The generalized fractional integrals (GFIs) defined above include standard fractional integrals, the left- and right-sided ones for kernels being power functions $k_{\alpha}(t, \tau)=k_{\alpha}(t-\tau)=(t-\tau)^{\alpha-1} / \Gamma(\alpha)$ and a suitable choice of parameters $P$. It is easy to check that the GFIs fulfill relations given below.

Property 1.2. Operator $K_{P}^{\alpha}$ satisfies the following formula

$$
K_{P}^{\alpha} f(t)=p K_{P_{1}}^{\alpha} f(t)+q K_{P_{2}}^{\alpha} f(t),
$$

where $P=(a, t, b, p, q), P_{1}=(a, t, b, 1,0), P_{2}=(a, t, b, 0,1)$.

Operator $K_{P}^{\alpha}$ satisfies the following integration by parts formula

$$
\int_{a}^{b} g(t) K_{P}^{\alpha} f(t) d t=\int_{a}^{b} f(t) K_{P^{*}}^{\alpha} f(t) d t,
$$

where $P=(a, t, b, p, q), P^{*}=(a, t, b, q, p)$.

In our considerations we shall investigate reflection symmetry properties of GFDs. In the finite interval $[\mathrm{a}, \mathrm{b}]$ the reflections operator assigns function at point $t$ its value at point $a+b-t$.

Definition 1.3. We define the reflection operator $Q$ in the interval $[a, b]$ as follows

$$
(Q f)(t)=f(a+b-t) .
$$


Assuming that the kernel determining a given fractional integral operator is invariant with respect to translations we obtain a simple relation between reflections and GFIs.

Property 1.4. If $k_{\alpha}(t, \tau)=k_{\alpha}(t-\tau)$, then the $K_{P}^{\alpha}$ and $Q$ operators satisfy the following identity

$$
Q K_{P}^{\alpha} f(t)=K_{P^{*}}^{\alpha} Q f(t),
$$

where parameters $P=(a, t, b, p, q), P^{*}=(a, t, b, q, p)$.

Now, using the above integrals we define the Riemann-Liouville and Caputo type generalized fractional derivatives.

Definition 1.5. The GFD of Riemann-Liouville type $A_{P}^{\alpha}$ and of Caputo type $B_{P}^{\alpha}$ are defined as follows:

$$
\begin{aligned}
& A_{P}^{\alpha} f(t)=D^{n} K_{P}^{n-\alpha} f(t) \\
& B_{P}^{\alpha} f(t)=K_{P}^{n-\alpha} D^{n} f(t),
\end{aligned}
$$

where $n-1<\alpha<n$ and $P=(a, t, b, p, q)$.

Both the $A_{P}^{\alpha}$ and $B_{P}^{\alpha}$ derivatives have well-defined commutation relations with respect to the reflection operator $Q$. These relations result from Property 1.4 for GFIs and properties of the integer order derivatives with respect to reflections.

Property 1.6. If $k_{\alpha}(t, \tau)=k_{\alpha}(t-\tau)$, then the operators $A_{P}^{\alpha}, B_{P}^{\alpha}$ and $Q$ satisfy the following identities

$$
\begin{aligned}
\mathrm{Q} A_{P}^{\alpha} f(t) & =(-1)^{n} A_{P^{*}}^{\alpha} Q f(t) \\
\mathrm{Q} B_{P}^{\alpha} f(t) & =(-1)^{n} B_{P^{*}}^{\alpha} Q f(t),
\end{aligned}
$$

where $P=(a, t, b, p, q), P^{*}=(a, t, b, q, p)$.

Now we define the symmetric and anti-symmetric GFIs of order $n-\alpha$ in the interval $[a, b]$ which are special cases of GFIs given in Definition 1.1. We shall apply them further in the construction of the symmetric and anti-symmetric derivatives.

Definition 1.7. The operators $K_{[a, b]}^{n-\alpha}$ and $\bar{K}_{[a, b]}^{n-\alpha}$ are given by the formulas

$$
\begin{gathered}
K_{[a, b]}^{n-\alpha} f(t)=K_{P_{1}}^{n-\alpha} f(t)+K_{P_{2}}^{n-\alpha} f(t)=K_{(a, t, b, 1,1)}^{n-\alpha} \\
\bar{K}_{[a, b]}^{n-\alpha} f(t)=K_{P_{1}}^{n-\alpha} f(t)-K_{P_{2}}^{n-\alpha} f(t)=K_{(a, t, b, 1,-1)}^{n-\alpha},
\end{gathered}
$$

where $P_{1}=(a, t, b, 1,0), P_{2}=(a, t, b, 0,1)$.

The above integrals are the non-local component of the symmetric and antisymmetric fractional derivatives over interval $[a, b]$. 
Definition 1.8. The symmetric $A_{[a, b]}^{\alpha}, B_{[a, b]}^{\alpha}$ and anti-symmetric $\bar{A}_{[a, b]}^{\alpha}, \bar{B}_{[a, b]}^{\alpha}$ derivatives of order $\alpha \in(n-1, n)$ in interval [a,b] are given by the formulas below:

$$
\begin{aligned}
A_{[a, b]}^{\alpha} f(t) & =D^{n} K_{[a, b]}^{n-\alpha} f(t) \\
\bar{A}_{[a, b]}^{\alpha} f(t) & =D^{n} \bar{K}_{[a, b]}^{n-\alpha} f(t) \\
B_{[a, b]}^{\alpha} f(t) & =K_{[a, b]}^{n-\alpha} D^{n} f(t) \\
\bar{B}_{[a, b]}^{\alpha} f(t) & =\bar{K}_{[a, b]}^{n-\alpha} D^{n} f(t) .
\end{aligned}
$$

The above derivatives can be expressed in terms of integer order derivatives and integrals being a generalization of Riesz potentials in finite interval [27].

Property 1.9. The symmetric $A_{[a, b]}^{\alpha}, B_{[a, b]}^{\alpha}$ and anti-symmetric $\bar{A}_{[a, b]}^{\alpha}, \bar{B}_{[a, b]}^{\alpha}$ derivatives are given by the following explicit formulas

$$
\begin{gathered}
A_{[a, b]}^{\alpha} f(t)=D^{n} \int_{a}^{b} k_{n-\alpha}(|t-\tau|) f(\tau) d \tau \\
B_{[a, b]}^{\alpha} f(t)=\int_{a}^{b} k_{n-\alpha}(|t-\tau|) D^{n} f(\tau) d \tau \\
\bar{A}_{[a, b]}^{\alpha} f(t)=D^{n} \int_{a}^{b} k_{n-\alpha}(|t-\tau|) \operatorname{sgn}(t-\tau) f(\tau) d \tau \\
\bar{B}_{[a, b]}^{\alpha} f(t)=\int_{a}^{b} k_{n-\alpha}(|t-\tau|) \operatorname{sgn}(t-\tau) D^{n} f(\tau) d \tau .
\end{gathered}
$$

Let us observe that the $A_{P}^{\alpha}$ and $B_{P}^{\alpha}$ derivatives can be rewritten using the notion of symmetric and anti-symmetric derivatives given in Definition 1.8, namely we have

$$
\begin{aligned}
& A_{P}^{\alpha}=\frac{p+q}{2} A_{[a, b]}^{\alpha}+\frac{p-q}{2} \bar{A}_{[a, b]}^{\alpha}, \\
& B_{P}^{\alpha}=\frac{p+q}{2} B_{[a, b]}^{\alpha}+\frac{p-q}{2} \bar{B}_{[a, b]}^{\alpha} .
\end{aligned}
$$

Taking into account relations (8), (9) we can derive the commutation rules for the symmetric and anti-symmetric generalized derivatives of order $\alpha \in(n-1, n)$ :

$$
\begin{array}{cc}
Q A_{[a, b]}^{\alpha}=(-1)^{n} A_{[a, b]}^{\alpha} Q & Q B_{[a, b]}^{\alpha}=(-1)^{n} B_{[a, b]}^{\alpha} Q \\
Q \bar{A}_{[a, b]}^{\alpha}=(-1)^{n+1} \bar{A}_{[a, b]}^{\alpha} Q & Q \bar{B}_{[a, b]}^{\alpha}=(-1)^{n+1} \bar{B}_{[a, b]}^{\alpha} Q .
\end{array}
$$

From the above relations it follows that the respective derivatives of order $\alpha \in(0,1)$ commute (anti-symmetric GFDs) or anti-commute (symmetric GFDs) with the reflection operator: 


$$
\begin{array}{cc}
Q A_{[a, b]}^{\alpha}=-A_{[a, b]}^{\alpha} Q & Q B_{[a, b]}^{\alpha}=-B_{[a, b]}^{\alpha} Q \\
Q \bar{A}_{[a, b]}^{\alpha}=\bar{A}_{[a, b]}^{\alpha} Q & Q \bar{B}_{[a, b]}^{\alpha}=\bar{B}_{[a, b]}^{\alpha} Q .
\end{array}
$$

In the paper we shall analyze reflections in a hierarchy of intervals $\left[a, b_{m}\right]$. First, we define the corresponding reflection operators.

Definition 1.10. Reflection operators $Q_{\left[a, b_{m}\right]}$ acting on arbitrary function $f$ determined in the interval $[a, b]$ are given as follows:

$$
Q_{\left[a, b_{m}\right]} f(t)=f\left(a+b_{m}-t\right)
$$

where

$$
b_{m}=\frac{\left(2^{m}-1\right) a+b}{2^{m}}, m=0,1, \ldots
$$

\section{Reflection symmetry of generalized fractional derivatives}

Now we shall investigate properties of GFDs acting on components of functions which we defined in papers $[1,2]$. Below, we recall this definition of $[J]$-projections/ components of arbitrary function.

Definition 2.1. Let $f$ be an arbitrary function determined in $[a, b]$ and vector $[J]=\left[j_{1}, \ldots, j_{m}\right]$ be an arbitrary $m$-component vector from set $\{0,1\}^{m}$. The following recursive formulas define the respective projections/components of function $f$ :

$$
\begin{gathered}
f_{[j]}(t)=\frac{1}{2}\left(1+(-1)^{j} Q_{[a, b]}\right) f(t) \\
f_{\left[J, j_{m+1}\right]}(t)= \begin{cases}\frac{1}{2}\left(1+(-1)^{j_{m+1}} Q_{\left[a, b_{m}\right]}\right) f_{[J]}(t) & t \leq b_{m} \\
\frac{1}{2} f_{[J]}(t) & t>b_{m},\end{cases}
\end{gathered}
$$

where $j, j_{m+1} \in\{0,1\}$.

Let us note that function $f$ can be split into the respective projections for any $m \in \mathbb{N}$

$$
\begin{aligned}
f_{[J]}(t) & =\sum_{j=0}^{1} f_{[J, j]}(t) \\
f(t) & =\sum_{[J]} f_{[J]}(t),
\end{aligned}
$$

where the summation in formula (31) is taken over all $m$-component vectors with coordinates in the two-element set $\{0,1\}$.

We illustrate the construction of $[J]$-projections with a simple example of function $f(t)=t^{2}$ for which we shall explicitly calculate some of its components in 
interval $[0,1]$. Here the reflection operator acts as follows on arbitrary function $g$ : $Q_{[0,1]} g(t)=g(1-t)$. Thus, for the monomial function $f$ given above we have:

$$
\begin{gathered}
f_{[0]}(t)=\frac{1}{2}\left(t^{2}+(1-t)^{2}\right)=t^{2}-t+\frac{1}{2} \\
f_{[1]}(t)=\frac{1}{2}\left(t^{2}-(1-t)^{2}\right)=t-\frac{1}{2} .
\end{gathered}
$$

Having split the function into projections $f_{[0]}$ and $f_{[1]}$, we can calculate the set of projections: $f_{[0,0]}, f_{[1,0]}, f_{[0,1]}$ and $f_{[1,1]}$. We apply the reflection operator in interval $[0,1 / 2]$ which acts on arbitrary function $g$ as $Q_{[0,1 / 2]} g(t)=g\left(\frac{1}{2}-t\right)$ and obtain:

$$
\begin{aligned}
& f_{[0,0]}(t)= \begin{cases}t^{2}-\frac{1}{2} t+\frac{3}{8} & t \leq 1 / 2 \\
\frac{1}{2} f_{[0]}(t) & t>1 / 2\end{cases} \\
& f_{[0,1]}(t)= \begin{cases}-\frac{1}{2} t+\frac{1}{8} & t \leq 1 / 2 \\
\frac{1}{2} f_{[0]}(t) & t>1 / 2\end{cases} \\
& f_{[1,0]}(t)= \begin{cases}-\frac{1}{4} & t \leq 1 / 2 \\
\frac{1}{2} f_{[1]}(t) & t>1 / 2\end{cases} \\
& f_{[1,1]}(t)= \begin{cases}\mathrm{t}-1 / 4 & t \leq 1 / 2 \\
\frac{1}{2} f_{[1]}(t) & t>1 / 2\end{cases}
\end{aligned}
$$

Let us point out that the set of $\left[j_{1}, j_{2}\right]$-projections can only be derived if $[j]-$ -projections are known. In general, the calculation of the $\left[J, j_{m+1}\right]$-projections requires that the $[J]$-projections should be previously determined. This is the reason why we call the formulas from Definition 2.1 the recursive ones.

In this paper we consider the properties of GFDs of order $\alpha \in(0,1)$. The general formulas for GFDs of arbitrary order and their application will be the subject of the subsequent paper.

Proposition 2.2. Let $f_{[j]}$ be the $[j]$-projection of function $f$ given by formula (28). The $A_{[a, b]}^{\alpha}$ and $B_{[a, b]}^{\alpha}$ operators in interval $[a, b]$ can be represented as follows

$$
\begin{aligned}
& A_{[a, b]}^{\alpha} f_{[j]}(t)=\left(1+(-1)^{1+j} Q_{[a, b]}\right) A_{\left[a, b_{1}\right]}^{\alpha} f_{[j]}(t) \\
& B_{[a, b]}^{\alpha} f_{[j]}(t)=\left(1+(-1)^{1+j} Q_{[a, b]}\right) B_{\left[a, b_{1}\right]}^{\alpha} f_{[j]}(t),
\end{aligned}
$$

where $b_{1}=\frac{a+b}{2}, \alpha \in(0,1)$.

Let $f_{[J]}$ be the $[J]$-projection of function $f$ given in equation (29) for vector $[J]=\left[j_{1}, \ldots, j_{m}\right]$ with components $j_{l} \in\{0,1\}, l=1, \ldots, m$. Its symmetric derivatives of order $\alpha \in(0,1)$ in the interval $[a, b]$ can be represented as follows: 


$$
\begin{aligned}
& A_{[a, b]}^{\alpha} f_{[J]}(t)=2^{m} \prod_{[J]} A_{\left[a, b_{m}\right]}^{\alpha} f_{[J]}(t) \\
& B_{[a, b]}^{\alpha} f_{[J]}(t)=2^{m} \prod_{[J]} B_{\left[a, b_{m}\right]}^{\alpha} f_{[J]}(t),
\end{aligned}
$$

where we denoted as $\prod_{[J]}$ the ordered product of the projection operators

$$
\prod_{[J]}:=2^{-m}\left(1+(-1)^{1+j_{1}} Q_{[a, b]}\right) \ldots\left(1+(-1)^{1+j_{m}} Q_{\left[a, b_{m}\right]}\right),
$$

$b_{m}$ are determined in formula (27) and derivatives in subintervals $\left[a, b_{m}\right]$ are explicitly given as follows for arbitrary function $g$ :

$$
\begin{aligned}
& A_{\left[a, b_{m}\right]}^{\alpha} g(t)=D \int_{a}^{b_{m}} k_{1-\alpha}(|t-\tau|) g(\tau) d \tau \\
& B_{\left[a, b_{m}\right]}^{\alpha} g(t)=\int_{a}^{b_{m}} k_{1-\alpha}(|t-\tau|) D g(\tau) d \tau .
\end{aligned}
$$

Proof. First, we check property (32) using the integration properties and reflection properties of the first order derivative

$$
\begin{aligned}
A_{[a, b]}^{\alpha} f_{[j]}(t) & =D K_{[a, b]}^{1-\alpha} f_{[j]}(t)=D \int_{a}^{b} k_{1-\alpha}(|t-\tau|) f_{[j]}(\tau) d \tau \\
& =D \int_{a}^{b_{1}} k_{1-\alpha}(|t-\tau|) f_{[j]}(\tau) d \tau+D \int_{b_{1}}^{b} k_{1-\alpha}(|t-\tau|) f_{[j]}(\tau) d \tau
\end{aligned}
$$

Next, we apply substitution $a+b-\tau=u$ and property of the projections with respect to the reflection in interval $[a, b]: f_{[j]}(a+b-u)=(-1)^{j} f_{[j]}(u)$ and obtain

$$
\begin{gathered}
A_{[a, b]}^{\alpha} f_{[j]}(t)=D \int_{a}^{b_{1}} k_{1-\alpha}(|t-\tau|) f_{[j]}(\tau) d \tau+ \\
+D \int_{a}^{b_{1}} k_{1-\alpha}(|t-a-b+u|) f_{[j]}(a+b-u) d u= \\
=\left(1+(-1)^{1+j} Q_{[a, b]}\right) D \int_{a}^{b_{1}} k_{1-\alpha}(|t-\tau|) f_{[j]}(\tau) d \tau= \\
=\left(1+(-1)^{1+j} Q_{[a, b]}\right) A_{\left[a, b_{1}\right]}^{\alpha} f_{[j]}(t) .
\end{gathered}
$$


Let us observe that equation (32) remains valid when we replace $b$ by $b_{m}$ and take $t \in\left[a, b_{m}\right]:$

$$
A_{\left[a, b_{m}\right]}^{\alpha} f_{\left[J, j_{m+1}\right]}(t)=\left(1+(-1)^{1+j_{m+1}} Q_{\left[a, b_{m}\right]}\right) A_{\left[a, b_{m+1}\right]}^{\alpha} f_{\left[J, j_{m+1}\right]}(t) .
$$

Using this observation, we can prove property (34) by means of the mathematical induction principle. From (32) and the definition of the projection operator (36), we obtain for the $\left[J, j_{m+1}\right]$-projections

$$
\begin{gathered}
A_{[a, b]}^{\alpha} f_{\left[J, j_{m+1}\right]}(t)=2^{m} \prod_{[J]} A_{\left[a, b_{m}\right]}^{\alpha} f_{\left[J, j_{m+1}\right]}(t)= \\
=2^{m} \prod_{[J]}\left(1+(-1)^{1+j_{m+1}} Q_{\left[a, b_{m}\right]}\right) A_{\left[a, b_{m+1}\right]}^{\alpha} f_{\left[J, j_{m+1}\right]}(t)= \\
=2^{m+1} \prod_{\left[J, j_{m+1}\right]} A_{\left[a, b_{m+1}\right]}^{\alpha} f_{\left[J, j_{m+1}\right]}(t)
\end{gathered}
$$

which proves formula (34) to be valid for arbitrary $m \in \mathbb{N}$. The calculations for properties (33) and (35) are similar.

Analogous proposition is valid for the anti-symmetric GFDs.

Proposition 2.3. Let $f_{[j]}$ be the $[j]$ projection of function $f$ given by formula (28). The $\bar{A}_{[a, b]}^{\alpha}$ and $\bar{B}_{[a, b]}^{\alpha}$ operators in interval $[a, b]$ can be represented as follows:

$$
\begin{aligned}
& \bar{A}_{[a, b]}^{\alpha} f_{[j]}(t)=\left(1+(-1)^{j} Q_{[a, b]}\right) \bar{A}_{\left[a, b_{1}\right]}^{\alpha} f_{[j]}(t) \\
& \bar{B}_{[a, b]}^{\alpha} f_{[j]}(t)=\left(1+(-1)^{j} Q_{[a, b]}\right) \bar{B}_{\left[a, b_{1}\right]}^{\alpha} f_{[j]}(t),
\end{aligned}
$$

where $b_{1}=\frac{a+b}{2}, \alpha \in(0,1)$.

Let $f_{[J]}$ be the $[J]$-projection of function $f$ given in equation (29) for vector $[J]=\left[j_{1}, \ldots, j_{m}\right]$ with components $j_{l} \in\{0,1\}, l=1, \ldots, m$. Its anti-symmetric derivatives of order $\alpha \in(0,1)$ in interval $[a, b]$ can be represented as follows

$$
\begin{aligned}
& \bar{A}_{[a, b]}^{\alpha} f_{[J]}(t)=2^{m} \prod_{[J]} \bar{A}_{\left[a, b_{m}\right]}^{\alpha} f_{[J]}(t) \\
& \bar{B}_{[a, b]}^{\alpha} f_{[J]}(t)=2^{m} \prod_{[J]} \bar{B}_{[a, m]}^{\alpha} f_{[J]}(t),
\end{aligned}
$$

where we denoted as $\prod_{[J]}$ the ordered product of the projection operators

$$
\prod_{[J]}:=2^{-m}\left(1+(-1)^{j_{1}} Q_{[a, b]}\right) \ldots\left(1+(-1)^{j_{m}} Q_{\left[a, b_{m}\right]}\right)
$$

$b_{m}$ are determined in formula (27) and derivatives in subintervals $\left[a, b_{m}\right]$ are explicitly given as follows for arbitrary function $g$ : 


$$
\begin{aligned}
& \bar{A}_{\left[a, b_{m}\right]}^{\alpha} g(t)=D \int_{a}^{b_{m}} k_{1-\alpha}(|t-\tau|) \operatorname{sgn}(t-\tau) g(\tau) d \tau \\
& \bar{B}_{\left[a, b_{m}\right]}^{\alpha} g(t)=\int_{a}^{b_{m}} k_{1-\alpha}(|t-\tau|) \operatorname{sgn}(t-\tau) D g(\tau) d \tau .
\end{aligned}
$$

\section{Conclusions}

In the paper we introduced the symmetric and anti-symmetric generalized fractional derivatives in interval $[a, b]$ and analyzed their properties with respect to the reflection mapping. Depending on the order of derivative, they commute or anticommute with the reflection operator. Next, we investigated how the GFDs in symmetric and anti-symmetric version act on components of functions with welldefined reflection symmetry. The main result presented in Propositions 2.2 and 2.3 are representation formulas for the $A_{[a, b]}^{\alpha}, B_{[a, b]}^{\alpha}$ and $\bar{A}_{[a, b]}^{\alpha}, \bar{B}_{[a, b]}^{\alpha}$ derivatives. We demonstrated explicitly that for each $m \in \mathbb{N}$, they can be written using only the $A_{\left[a, b_{m}\right]}^{\alpha}, B_{\left[a, b_{m}\right]}^{\alpha}$ or $\bar{A}_{\left[a, b_{m}\right]}^{\alpha}, \bar{B}_{\left[a, b_{m}\right]}^{\alpha}$ GFDs respectively. In the case of standard fractional calculus such properties of symmetric and anti-symmetric operators led to a localization of Euler-Lagrange equations [1,2]. Generalized fractional mechanics and the localization of its equations of motion will be the subject of our further investigations.

\section{References}

[1] Klimek M., Lupa M., On reflection symmetry in fractional mechanics, Scientific Research of the Institute of Mathematics and Computer Science 2011, 10, 109-121.

[2] Klimek M., On reflection symmetry and its application to the Euler-Lagrange equations in fractional mechanics, Phil. Trans. R. Soc. A to appear, 2013.

[3] Riewe F., Nonconservative Lagrangian and Hamiltonian mechanics, Phys. Rev. E 1996, 53, 1890$-1899$.

[4] Riewe F., Mechanics with fractional derivatives, Phys. Rev. E 1997, 55, 3581-3592.

[5] Agrawal O.P., Formulation of Euler-Lagrange equations for fractional variational problem, J. Math. Anal. Appl. 2002, 272, 368-379.

[6] Klimek M., Fractional sequential mechanics - models with symmetric fractional derivative, Czech. J. Phys. 2001, 51, 1348-1354.

[7] Klimek M., Lagrangean and Hamiltonian fractional sequential mechanics, Czech. J. Phys. 2002, $52,1247-1253$.

[8] Agrawal O.P., Fractional variational calculus in terms of Riesz fractional derivatives, J. Phys. A: Math. Theor. 2007, 40, 6287-6303.

[9] Almeida R., Torres D.F.M., Necessary and sufficient conditions for the fractional calculus of variations with Caputo derivatives, Commun. Nonlinear Sci. Numer. Simulat. 2011, 16, 1490-1500 . 
[10] Almeida R., Pooseh S., Torres D.F.M., Fractional variational problems depending on indefinite integrals, Nonlinear Anal. TMA 2012, 75, 1009-1025.

[11] Atanackovic T.M., Stankovic B., On a differential equation with left and right fractional fractional derivatives, Fract. Calc. Appl. Anal. 2007, 10, 138-150.

[12] Atanackovic T.M., Konjik P., Pilipovic S., Variational problems with fractional derivatives: Euler-Lagrange equations, J. Phys. A: Math. Theor. 2008, 41, 095201.

[13] Baleanu D., Avkar T., Lagrangians with linear velocities within Riemann-Liouville fractional derivatives, Nuovo Cim. B 2004, 119, 73-79.

[14] Baleanu D., Muslish S., Lagrangian formulation of classical fields within Riemann-Liouville fractional derivatives, Physica Scripta 2005 ,72, 119-121.

[15] Baleanu D., Agrawal O.P., Fractional Hamilton formalism within Caputo's derivative, Czech. J. Phys. 2006, 56, 1087-1092.

[16] Baleanu D., Trujillo J.J., A new method of finding the fractional Euler-Lagrange and Hamilton equations within fractional Caputo derivatives, Commun. Nonlinear Sci. Numer. Simulat. 2010 $15,1111-1115$.

[17] Cresson J., Fractional embedding of differential operators and Lagrangian systems, J. Math. Phys. 2007, 48, 033504.

[18] Klimek M., Lagrangian fractional mechanics - a non-commutative approach, Czech. J. Phys. $2005,55,1447-1454$.

[19] Malinowska A.B., Torres D.F.M., Generalized natural boundary conditions for fractional variational problems in terms of Caputo derivative, Comput. Math. Appl. 2010, 59, 3110-3116.

[20] Odzijewicz T., Malinowska A.B., Torres D.F.M., Fractional variational calculus with classical and combined Caputo derivatives, Nonlinear Anal. TMA 2012, 75, 1507-1515.

[21] Agrawal O.P., Generalized variational problems and Euler-Lagrange equations, Comput. Math. Appl. 2010, 59, 1852-1864.

[22] Kilbas A.A., Saigo M., Generalized Mittag-Leffler function and generalized fractional calculus operators, Integral Transform. Spec. Func. 2004,15, 31-49.

[23] Srivastava H.M., Tomovski Z., Fractional calculus with an integral operator containing a generalized Mittag-Leffler function in the kernel, Appl. Math. Comput. 2009, 211, 198-210.

[24] Tomovski Z., Hilfer R., Srivastava H.M., Fractional and operational calculus with generalized fractional derivative operators and Mittag-Leffler type functions, Integral Transform. Spec. Func. 2010, 21, 797-814.

[25] Tomovski Z., Sandev T., Fractional wave equation with a frictional memory kernel of Mittag-Leffler type, Appl. Math. Comput. 2012, 218, 10022-10031.

[26] Tomovski Z., Sandev T., Effects of a fractional friction with power-law memory kernel on string vibrations, Comp. Math. Appl. 2011, 62, 1554-1561.

[27] Samko S.G., Kilbas A.A., Marichev O.I., Fractional Integrals and Derivatives. Theory and Applications, Gordon \& Breach Science Publ., New York 1993. 